\title{
Changes in Health Behaviors and the Trajectory of Body Mass Index among Older Japanese: A 19-year Longitudinal
}

\section{Study}

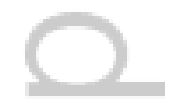

Hiroshi Murayama ${ }^{\mathrm{a}, b^{*}}$, Jersey Liang ${ }^{\mathrm{c}}$, Benjamin A. Shaw ${ }^{\mathrm{d}}$, Anda Botoseneanu ${ }^{\mathrm{e}, \mathrm{f},}$, Erika Kobayashi $^{\mathrm{b}}$, Taro Fukaya ${ }^{\mathrm{b}}$, and Shoji Shinkai ${ }^{\mathrm{b}}$

${ }^{a}$ Institute of Gerontology, The University of Tokyo

${ }^{\mathrm{b}}$ Tokyo Metropolitan Institute of Gerontology

${ }^{c}$ Department of Health Management and Policy, University of Michigan School of Public Health

${ }^{\mathrm{d}}$ Department of Health Policy, Management and Behavior, School of Public Health, State University of New York at Albany

${ }^{\mathrm{e}}$ Department of Health and Human Services, University of Michigan

${ }^{\mathrm{f}}$ Institute of Gerontology, University of Michigan

*Corresponding author: Hiroshi Murayama

7-3-1 Hongo, Bunkyo-ku, Tokyo 113-8656, JAPAN

Tel: +81-3-5841-0232, Fax: +81-3-5841-1662, e-mail: murayama@iog.u-tokyo.ac.jp

This is the author manuscript accepted for publication and has undergone full peer review but has not been through the copyediting, typesetting, pagination and proofreading process, which may lead to differences between this version and the Version of Record. Please cite this article as doi: $10.1111 /$ ggi.13008

This article is protected by copyright. All rights reserved. 
Running title: Health behaviors and BMI trajectory

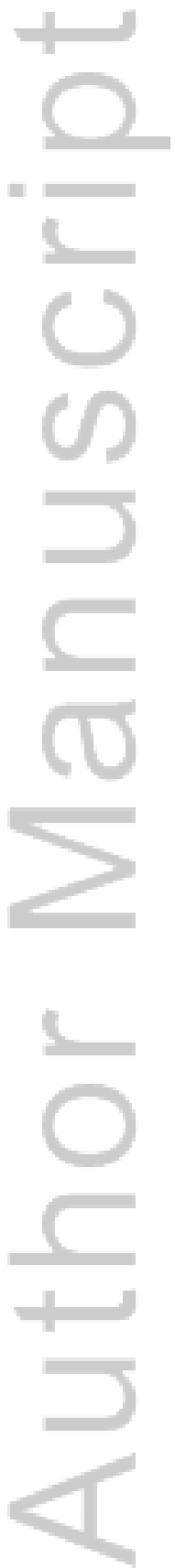

This article is protected by copyright. All rights reserved. 


\section{ABSTRACT}

Aim: Although the modification of lifestyle factors may facilitate weight control, the effects of health behaviors on trajectory of body weight among older adults have been understudied. We examined the effect of changes in smoking, alcohol use and physical activity on the long-term trajectory of body mass index (BMI) among older Japanese.

Methods: Data came from a national sample of 4,869 Japanese adults aged 60 and older at baseline, with up to seven repeated observations over a period of 19 years (19872006). Hierarchical linear modeling was used to analyze the intrapersonal and interpersonal differences in BMI trajectory.

Results: Average BMI among older Japanese was 22.3 at baseline and decreased with an accelerating rate over time. Smoking was significantly associated with lower BMI over time, while smoking cessation was associated with higher BMI. Drinking and physical activity were not associated with BMI. We found significant interactions between age and smoking status, and between gender and physical activity, on BMI trajectory: the association between smoking and lower BMI was stronger in younger participants compared with older participants. The association between physical activity and higher BMI was more pronounced among males compared with females.

Conclusion: Our findings yield important new information regarding the complex 
dynamics underlying the linkage between lifestyles factors and BMI trajectory among older Japanese, and suggest that there may be cross-cultural differences in these linkages.

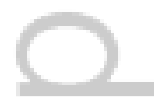

Keywords: body mass index; health behavior; hierarchical linear modeling; Japan; trajectory

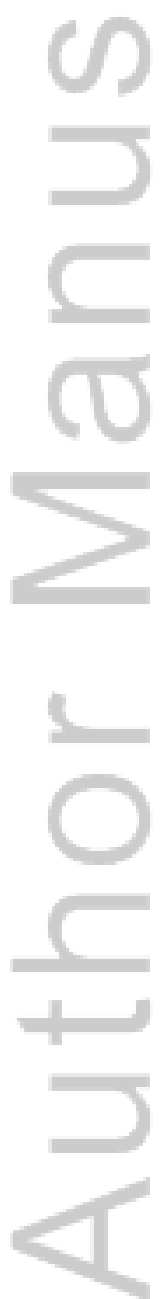

This article is protected by copyright. All rights reserved. 


\section{INTRODUCTION}

Weight change has been shown to predict mortality risk. Weight loss, in particular unintentional weight loss, has been associated with an increased risk of all-cause mortality, while findings on the relationship between weight gain and mortality have been mixed in older populations. ${ }^{1-5}$ Most studies have defined weight change on the basis of body mass index (BMI) measured at two points in time over a relatively short interval. However, because body weight can fluctuate substantially over the adult life course, ${ }^{6-8}$ analyzing weight changes across multiple points in time (i.e., the trajectory of BMI) may be important in understanding weight control and management of weight-related conditions in late life.

The modification of lifestyle factors, such as smoking, ${ }^{9-11}$ alcohol use, ${ }^{12,13}$ and physical activity ${ }^{14}$ may facilitate weight control in middle- and older age. However, to our knowledge, there has been only one study exploring the relationship between changes in lifestyle factors and the trajectory of BMI in this age group. A 14-year longitudinal study of U.S. adults aged 51-61 at the baseline, ${ }^{15}$ revealed distinct patterns of stability and changes (initiation and cessation) in smoking, alcohol use and physical activity, and a complex association with the trajectory of BMI. In particular, smoking and physical activity were associated with descending BMI trajectory, while cessation 
of smoking and physical activity were associated with ascending BMI trajectory.

However, populations in Western countries differ significantly from those in non-Western countries, both in terms of body weight and lifestyle factors. ${ }^{16,17}$ Moreover, education and household income appear to be associated with BMI among older Japanese $^{18}$ in different ways from those among older Americans. ${ }^{6}$ In addition, the proportions of non-smokers and people who engage in high-intensity activity among Japanese adults are lower than those among U.S. adults. ${ }^{19,20}$ Because most research on BMI trajectories among older people is based on data derived from Western societies, particularly the U.S., it is important to explore if the relationship between lifestyle factors and BMI trajectories differ among older Japanese. This could inform the design of health policies and interventions aimed at promoting and maintaining body-weight in late life, particularly in Asian populations.

Furthermore, in light of the age and gender differences in the prevalence of overweight/obesity, smoking, alcohol consumption, and daily physical activity among older Japanese, ${ }^{16}$ it is also important to explore whether the association between lifestyle factors and BMI trajectory vary according to age and gender. This knowledge may contribute to the design of effective clinical and public health interventions aimed at maintaining healthy weight among older adults. 
The purpose of this study is to examine the association between health behaviors (smoking, alcohol use, and physical activity) and BMI within the context of the long-term trajectory of body weight among older Japanese. In addition, we analyzed age and gender variations in the associations between health behaviors and BMI trajectory. Because health behaviors can vary over time within individuals, ${ }^{21,22}$ we specified health behaviors as time-varying covariates to more accurately describe the actual change in behavioral patterns.

\section{METHODS}

\section{Sample and procedures}

Data came from the National Survey of the Japanese Elderly (NSJE), a 7-wave (1987, 1990, 1993, 1996, 1999, 2002, and 2006) longitudinal panel of 4,869 Japanese, 60 years of age and older, with 16,669 observations over 19 years (1987-2006). This sampling procedure has been previously described in detail. ${ }^{18,22}$ This survey began with $\underline{\text { 2,200 respondents aged } 60 \text { and over in } 1987 \text { (wave 1). The sample was subsequently }}$ supplemented in 1990 (wave 2; n=580) and 1996 (wave 4; $\mathrm{n}=1,210$ ). An additional sample of those 70 years of age and older was added in 1999 (wave 5; $n=2,000$ ). The 
number of study participants at each wave and the number of observations of participant individuals by entry wave were shown in supporting information 1 and 2 . Trained investigators visited the respondents' homes, and face-to-face interviews employing a structured questionnaire were used to collect the data. The study was approved by the Institutional Review Board of the Tokyo Metropolitan Institute of Gerontology.

\section{Measures}

$B M I$

Respondents' self-reported height and weight were recorded at each wave. The dependent variable BMI was calculated according to the formula: BMI=weight $(\mathrm{kg}) /$ height $(\mathrm{m})^{2}$.

\section{Health behaviors status and changes}

Current smoking, alcohol use, and physical activity were recorded at every wave (time-varying variables). With regard to smoking and alcohol use, participants were classified into " $0=$ non-users" or " $1=$ users". For physical activity, we used two items: regular exercise and walking over $1 \mathrm{~km}$ in a daily life (" $0=$ rarely/never" or "1=often/sometimes"). Generally, physical activity includes exercise which is planned, 
structured, repetitive, and purposeful in the sense that the improvement or maintenance of one or more components of physical fitness is the objective, and other activities which involve bodily movement and are done as part of playing, working, active transportation, house chores and recreational activities. ${ }^{23}$ Therefore, we set these two items, and classified participants into three categories at each time point: " $0=$ doing neither activities (non-active)," "1=doing either (moderately active)," or " $2=$ doing both (active)."

Changes in each behavior between two adjacent waves were captured by a difference score (i.e., difference between current $\left[t_{i}\right]$ and previous observation $\left[t_{i}-1\right]$ ) calculated for each wave. For smoking and alcohol use, three categories were created (i.e., $-1=$ cessation, $0=$ no change, $1=$ initiation). For physical activity, five categories were possible, ranging from -2 to 2 ; however, for consistency with analyses and interpretation on smoking and alcohol use, we re-classified them into three categories: $-1=$ cessation (including all levels of reduction]), $0=$ no change, $1=$ initiation (including all levels of increase). This approach is consistent with previous research, which used the same analytic framework for older Americans. ${ }^{15}$

\section{Covariates}

This article is protected by copyright. All rights reserved. 
Age, gender, and education were used as time-constant variables recorded at baseline. Additional time-varying covariates included: marital status, working status, annual household income, and three measures assessing health status. Household income was assessed by using five categories: "1=less than 1.2 million yen per year," " $2=1.2-3$ million yen per year," " $3=3-5$ million yen per year," " $4=5-10$ million yen per year," and " $5=$ more than 10 million yen per year." Three measures of health status were included: 1) chronic conditions, measured as a count of 16 chronic diseases (e.g., cancer, high blood pressure, heart conditions, diabetes, stroke); 2) self-rated health, measured by a three-item composite with scores ranging from 3 to 15 (Cronbach's alpha $=0.85)$, and a high score indicates poorer health; and 3) functional status, measured as a sum of difficulties with six activities of daily living and five instrumental activities of daily living. With regard to functional status, all items were scored by either a 4- or 5-point scale, and with appropriate adjustment, scores for this composite ranged from 11.5 to 55 (Cronbach's alpha=0.92); a higher score reflects greater functional disability.

\section{Data Analysis}

Hierarchical linear models (HLM) were used to chart the trajectory of BMI from

1987 to 2006. HLMs are well suited for studies of individual changes over time, using 
repeated measures of a construct to estimate a growth trajectory defined by intercept (i.e., level during the observation) and slope (i.e., rate of change, such as rate of linear change and rate of acceleration). The analytical strategy employed a series of time-based models sequentially adjusted to control for interpersonal age-at-baseline and sociodemographic differences. The year when a respondent was first interviewed was used as the baseline for each individual. Time (i.e., the distance of assessment from the baseline) was centered on its grand mean (5.43 years) to minimize the possibility of multicollinearity when estimating nonlinear time functions. All models were fitted by using HLM 7.

BMI at a given time (Level 1 equations) was specified as a function of time (i.e., distance from baseline year) and time-varying covariates (e.g., smoking, alcohol use, physical activity, marital status, working status, household income, and health status). To ensure a clear time sequencing between dependent and independent variables, each time-varying covariate was represented by two distinct measures: a lagged measure (i.e., observation from the previous wave $\left[t_{i}-1\right]$ ) and a change term (i.e., difference between current $\left[t_{i}\right]$ and previous observation $\left.\left[t_{i}-1\right]\right)$. Moreover, two interaction terms were created for each health behavior and added in the Level 1 equations to assess whether the strength of the association between the lagged status or the change score and BMI 
trajectory varied by age and gender (e.g., [lagged smoking $\times$ age $]$ and $[\Delta$ smoking $\times$ age]). Interpersonal variations were evaluated by including time-constant covariates, such as age-at-baseline, gender, education, and baseline BMI level in the Level 2 equations.

To adjust for possible selection bias, we included mortality and attrition due to non-response in the Level 2 equation. To minimize missing data due to item non-response, multiple imputation was undertaken. In particular, three complete data sets were imputed with the NORM software. ${ }^{24}$ The modeling strategy involved the following five steps: Model 1 included health behaviors (both lagged and change terms) and time-constant covariates; Model 2 added marital status, working status, household income, and health status; Model 3 was additionally adjusted for baseline BMI; Models 4 and 5 added age and gender interaction terms, to evaluate age and gender differences in the association between health behaviors and BMI trajectories. Supporting information 3 provides more detailed descriptions of the analytic strategy.

\section{RESULTS}

Table 1 presents descriptive statistics for the study participants at the baseline. 
The average age of the respondents was 69.85 and women accounted for $55.3 \%$ of the sample. The proportions of people who died during the study and who dropped out for $\underline{\text { reasons other than mortality and did not return in subsequent waves were } 36.7 \% \text { and }}$ 21.0\%, respectively. The average BMI was 22.26. The proportions of respondents who smoked and drunk were $24.5 \%$ and $40.3 \%$ respectively. In addition, $38.6 \%, 33.6 \%$, and $27.9 \%$ of the respondents were active, moderate, and non-active in physical activities. Table 2 shows time-varying characteristics by wave, including BMI, smoking, alcohol use, and physical activity. Average BMI during the 19-year observation was 22.16.

<Insert Tables 1 and 2>

Table 3 shows the results of the multilevel linear regression model. To depict the trajectory of BMI between 1987 and 2006, we evaluated linear, quadratic, and cubic models and chose the most appropriate one on the basis of the statistical significance of higher-order fixed effects estimates. As the cubic coefficient was not statistically significant, the change in BMI was best described by a quadratic function. The unconditional model indicated that BMI decreased with a quadratic curve (from 22.3 to 21.2 over the 19 years of observation).

In Model 1, smoking was significantly associated with lower BMI over time $(b=-0.722)$. Moreover, its cessation were significantly associated higher BMI 
$(b=-0.431)$. While drinking and physically activeness were not associated with BMI in Model 1, initiations/increases of these health behaviors were associated with higher BMI over time $(b=0.154$ for alcohol use and 0.067 for physical activity). When adjusting for both lagged status and change terms of covariates in Model 2, the effects of initiation/increase of alcohol use and physical activity became non-significant. These estimates remained non-significant in Model 3 (adjusting for baseline BMI). However, the effects of smoking and its change on BMI remained significant in Models 2 and 3 (e.g., $b=-0.358$ and -0.281 in Model 3).

Models 4 and 5 examined age and gender differences in the association between health behaviors and BMI. Model 4 showed a significant interaction between age and lagged smoking status on BMI $(b=0.040)$, indicating that smokers had lower BMI than non-smokers in younger cohorts, but this effect became smaller in older cohorts. In Model 5, significant interactions between gender and physical activity, and between gender and the initiation of physical activity, on BMI were observed $(b=-0.129$ and -0.144). This demonstrated that the effects of physical activity and initiation/increase of physical activity on higher BMI were greater among males compared to females. In order to confirm these interactions, we performed gender-stratified analysis, and found significant associations between physical activity 
(of both lagged and change terms) and higher BMI in the male subsample, but not in the female subsample (see supporting information 4).

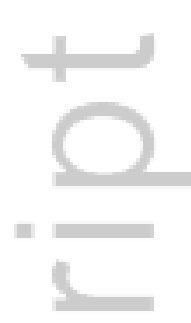

$<$ Insert Tables 3>

\section{DISCUSSION}

This study examined the association between changes in smoking, alcohol use, and physical activity and the trajectory of BMI among older Japanese over the course of a 19-year period. To our knowledge, this is the first study to explore these effects in the older Japanese population. Our findings suggest lifestyle interventions as possible clinical and public health tools aimed at promoting and maintaining healthy body-weight in older age.

Smoking was associated with lower BMI, while its cessation was associated with higher BMI. Nicotine intake is accompanied by a decreased consumption of sweet-tasting high caloric foods. ${ }^{25}$ In fact, cross-sectional studies reported the relationship between smoking and lower body weight, ${ }^{9,10}$ including a study in Japan. ${ }^{11}$ It is also suggested that smoking quitters tend to gain weight. ${ }^{26}$ In addition, our results concerning the effect of smoking on BMI trajectory are consistent with previous 
research focusing on BMI trajectory among Americans aged 51-61 at baseline. ${ }^{15}$ However, the effects of smoking (i.e., lagged smoking status and changes in smoking) seemed to be smaller in older Japanese than those in older Americans (e.g., compared to non-smokers, 0.36-0.73 BMI units lower for smokers in our samples, but 1.05-1.38 BMI units lower in older Americans ${ }^{15}$ ). Of course, because the age of the sample, sample size and covariates adjusted in the models were different between these two cohorts, direct comparisons of these values should be made with caution. However, if this difference is valid, it might be due to the difference in the BMI level between two older populations. ${ }^{16,17}$ That is, as average BMI among older Americans is higher than that among older Japanese, the effects of smoking on decreases of body weight might be greater.

Additionally, a significant interaction between age and lagged smoking status on BMI was observed in Model 4. This suggests that smoking was associated with lower BMI than non-smoking in younger cohorts, but this association became smaller in older cohorts. Among Americans, the difference in BMI between smokers and non-smokers was also greater in young cohorts than among middle-aged cohorts. ${ }^{27}$ The sample of this study was limited to older ages, but the trend of age differences in the linkage between smoking and body weight seems similar. Previous research has 
reported that the prevalence of cigarette smoking decreases with age in older Japanese. ${ }^{22}$ Therefore, the effect of smoking on BMI might be attenuated with age.

Initiation of both alcohol use and physical activity were associated with higher BMI in Model 1; however, these associations became non-significant after adjusting for covariates and baseline BMI in Models 2 and 3. In the fully adjusted model (Model 3), higher household income and better self-rated health were significantly related to higher BMI over time. These factors might be proxies of alcohol use and physical activity. Indeed, drinking and physically activity were both significantly associated with higher income and better self-rated health in our samples (see supporting information 5). This implies that people who have high income and/or good health condition possibly drink and become physically active. Conversely, people with low income and/or poor health condition might or must abstain from drinking and physical activity.

Previous research has reported that increases in physical activity and decreases in sedentary time are related to weight loss among U.S. adults with obesity, ${ }^{28}$ which is consistent with the U.S. study exploring the association between lifestyles and BMI trajectory. ${ }^{15}$ However, because the proportions of overweight and obesity are lower in older Japanese compared to those in older American, ${ }^{16,17}$ the effect of physical activity on BMI might be smaller and non-significant in our samples. On the other hand, we 
found significant interactions between gender and physical activity (of both lagged and change terms) on BMI in Model 5. This indicates that the effects of physical activity and initiation/increase of physical activity on higher BMI were greater among males compared to females. A similar gender-differential pattern of association has been reported in other epidemiological studies. ${ }^{14}$ Physical activity can increase muscle mass of older people in both genders. ${ }^{29}$ However, as females have more fat mass than males, ${ }^{30}$ physical activity can easily promote fat-burning in female. This means that the fat-burning effect might be greater among females than in males; as such, physical activity could trigger an increase of muscle mass and a decrease of fat mass in female, thus obscuring a change in BMI.

This research has several limitations. First, we used self-reported height and weight, because the objectively-measured indicators were not available. People tend to over-report their height and underreport their weight, ${ }^{31}$ thus the level of BMI may be underestimated. However, if the level of under/over-reporting is relatively constant over time, the estimation of BMI trajectories over time should not be biased by mis-reporting. Second, because our sample consisted of only respondents aged 60 and over, it is unclear BMI status and changes in young and middle age moderate the effects of health behaviors on the BMI trajectory. Future studies are needed to evaluate the association 
between health behaviors and BMI trajectory over the life course. Third, during the period of observation (1987-2006), several health policies to promote healthy lifestyles were initiated in Japan, such as the "Active 80 Health Plan" in 1988 and "Healthy Japan 21 " in 2000. Data limitations did not allow us to assess whether the health behaviors examined here and/or the trajectory of BMI may have been influenced by these policy changes. Fourth, although we treated the BMI trajectory, we did not use any nutritional and dietary indicators in the analysis. Future research should consider these effects on BMI trajectory.

A major strength of this research lies in the longitudinal data derived from a national probability sample of older Japanese over a 19-year period. This study also considered time-varying lifestyle factors (i.e., the lagged and change terms) as independent variables. This research yields important new information regarding the complex dynamics underlying the linkage between lifestyles factors and BMI trajectory among older Japanese. In addition, as we mentioned above, there are several differences in health behaviors between Western countries such as the U.S. and non-Western countries such as Japan. However, compared to a similar study among older Americans, ${ }^{15}$ we found both similarities and dissimilarities between the U.S. and Japan with regard to the linkage between lifestyle factors and BMI trajectory in old age. Our 
results suggest that there are cross-cultural differences in the linkage between lifestyle factors and BMI. Further research is clearly needed not only to replicate our observations but also to further specify the nature of the cross-cultural variations in the linkage.

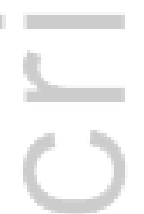

\section{Funding}

This work was supported by the National Institute on Aging (Grant No. R01 AG031109 and Grant No. P60 AG024824) at the National Institutes of Health.

\section{Disclosure statement}

The authors declare no conflict of interest.

This article is protected by copyright. All rights reserved. 


\section{REFERENCES}

1. Corrada MM, Kawas CH, Mozaffar F, Paganini-Hill A. Association of body mass index and weight change with all-cause mortality in the elderly. Am $J$ Epidemiol. 2006;163:938-949.

2." Dey DK, Rothenberg E, Sundh V, Bosaeus I, Steen B. Body mass index, weight change and mortality in the elderly. A $15 \mathrm{y}$ longitudinal population study of 70 y olds. Eur J Clin Nutr. 2001;55:482-492.

3. Nanri A, Mizoue T, Takahashi Y, et al. Weight change and all-cause, cancer and cardiovascular disease mortality in Japanese men and women: the Japan Public Health Center-Based Prospective Study. Int J Obes. 2010;34:348-356.

4. Newman AB, Yanez D, Harris $T$, et al. Weight change in old age and its association with mortality. J Am Geriatr Soc. 2001;49:1309-1318.

5. Somes GW, Kritchevsky SB, Shorr RI, Pahor M, Applegate WB. Body mass index, weight change, and death in older adults: the systolic hypertension in the elderly program. Am J Epidemiol. 2002;156:132-138.

6. Botoseneanu A, Liang J. Social stratification of body weight trajectory in middle-age and older americans: results from a 14-year longitudinal study. $J$

Aging Health. 2011;23:454-480.

This article is protected by copyright. All rights reserved. 
7. Clarke P, O'Malley PM, Johnston LD, Schulenberg JE. Social disparities in BMI trajectories across adulthood by gender, race/ethnicity and lifetime socio-economic position: 1986-2004. Int J Epidemiol. 2009;38:499-509.

8. Fuemmeler BF, Yang CM, Costanzo P, et al. Parenting styles and body mass index trajectories from adolescence to adulthood. Health Psychol. 2012;31:441-449.

9. Bish CL, Blanck HM, Serdula MK, Marcus M, Kohl HW 3rd, Khan LK. Diet and physical activity behaviors among Americans trying to lose weight: 2000

Behavioral Risk Factor Surveillance System. Obes Res. 2005;13:596-607.

10. Kruger J, Ham SA, Prohaska TR. Behavioral risk factors associated with overweight and obesity among older adults: the 2005 National Health Interview Survey. Prev Chronic Dis. 2009;6:A14.

11. Ishizaki M, Morikawa Y, Nakagawa H, Honda R, Kawakami N, Haratani T, et al. The influence of work characteristics on body mass index and waist to hip ratio in Japanese employees. Ind Health. 2004;42:41-49.

12. Wannamethee SG, Field AE, Colditz GA, Rimm EB. Alcohol intake and 8-year weight gain in women: a prospective study. Obes Res. 2004;12:1386-1396.

13. Wannamethee SG, Shaper AG. Alcohol, body weight, and weight gain in 
middle-aged men. Am J Clin Nutr. 2003;77:1312-1317.

14. Liebman M, Pelican S, Moore SA, et al. Dietary intake, eating behavior, and physical activity-related determinants of high body mass index in rural communities in Wyoming, Montana, and Idaho. International journal of obesity and related metabolic disorders. Int JObes Metab Disord. 2003;27:684-692.

15. Botoseneanu A, Liang J. The effect of stability and change in health behaviors on trajectories of body mass index in older Americans: a 14-year longitudinal study. J Gerontol A Biol Sci Med Sci. 2011;67:1075-1084.

16. Ministry of Health, Labour and Welfare. The National Health and Nutrition Survey in Japan, 2013. Tokyo: Ministry of Health, Labour and Welfare; 2015.

17. Schoenborn CA, Adams PF, Peregoy JA. Health behaviors of adults: United States, 2008-2010. Vital Health Stat. 2013;10(257).

18. Murayama H, Liang J, Bennett JM, et al. Socioeconomic status and the trajectory of body mass index among older Japanese: a nationwide cohort study of 1987-2006. J Gerontol B Psychol Sci Soc Sci. 2016;71:378-388.

19. Organization for Economic Cooperation and Development. OECD Health Statistics 2015 [Internet]. Paris: Organization for Economic Cooperation and Development; 2015 [Cited 1 Nov 2015]. Available from: 
http://www.oecd.org/els/health-systems/health-data.htm

20. Bauman A, Bull F, Chey $\mathrm{T}$, et al. The international prevalence study on physical activity: results from 20 countries. Int J Behav Nutr Phys Act. $2009 ; 6: 21$

21. Shaw BA, Agahi N, Krause N. Are changes in financial strain associated with changes in alcohol use and smoking among older adults? J Stud Alcohol Drugs. 2011;72:917-925.

22. Murayama H, Bennett JM, Shaw BA, et al. Does social support buffer the effect of financial strain on the trajectory of smoking in older Japanese? a 19-year longitudinal study. J Gerontol B Psychol Sci Soc Sci. 2015;70:367-376.

23. World Health Organization. Physical activity [Internet]. Geneva: World Health Organization; 2016 [Cited 1 Dec 2016]. Available from: http://www.who.int/dietphysicalactivity/pa/en/

24. Schafer JL. Analysis of incomplete multivariate data. London: Chapman \& Hall; 1997.

25. Grunberg NE. The effects of nicotine and cigarette smoking on food consumption and taste preferences. Addict Behav. 1982;7:317-331.

26. Chiolero A, Faeh D, Paccaud F, Cornuz J. Consequences of smoking for body 
weight, body fat distribution, and insulin resistance. Am J Clin Nutr. 2008;87:801-809.

27. Jacobs DR Jr., Gottenborg S. Smoking and weight: the Minnesota Lipid Research Clinic. Am J Public Health. 1981;71:391-396.

28. Pellegrini CA, Song J, Chang R, et al. Change in physical activity and sedentary time associated with 2-year weight loss in obese adults with osteoarthritis. J Phys Act Health. 2016;13:461-466.

29. Shephard RJ, Park H, Park S, Aoyagi Y. Objectively measured physical activity and progressive loss of lean tissue in older Japanese adults: longitudinal data from the Nakanojo study. J Am Geriatr Soc. 2013;61:1887-1893.

30. Kitamura I, Koda M, Otsuka R, Ando F, Shimokata H. Six-year longitudinal changes in body composition of middle-aged and elderly Japanese: age and sex differences in appendicular skeletal muscle mass. Geriatr Gerontol Int. $2014 ; 14: 354-361$

31. Gunnell D, Berney L, Holland P, et al. How accurately are height, weight and leg length reported by the elderly, and how closely are they related to measurements recorded in childhood? Int J Epidemiol. 2000;29:456-464. 


\section{List of supporting information}

\section{Supporting information 1}

The number of study participants at each wave

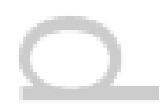

Supporting information 2

The number of observations by entry wave

Supporting information 3

The detailed description of data analysis

Supporting information 4

The effect of health behaviors on the trajectory of body mass index by gender (Based on

Model 3)

Supporting information 5

Household income and self-rated health by the status of alcohol use and physical activity

This article is protected by copyright. All rights reserved. 
Table 1. Baseline characteristics of the participants $(n=4,869)$

\begin{tabular}{|c|c|c|c|}
\hline & Total & Male & Female \\
\hline Female & 55.3 & - & - \\
\hline Age (years) & $69.85(7.20)$ & $69.41(7.10)$ & $70.21(7.27)$ \\
\hline Education (years) & $9.16(2.76)$ & $9.77(2.95)$ & $8.66(2.49)$ \\
\hline Married & 67.1 & 88.3 & 49.9 \\
\hline Current working & 31.7 & 44.5 & 21.2 \\
\hline Household income (range: $1-5$ ) & $2.87(1.10)$ & $3.07(1.07)$ & $2.71(1.10)$ \\
\hline Total condition (range: $0-16$ ) & $1.23(1.36)$ & $1.11(1.28)$ & $1.33(1.42)$ \\
\hline Self-rated health (range: $3-15$ ) & $7.69(2.81)$ & $7.41(2.81)$ & $7.92(2.80)$ \\
\hline Functional status (range: $11.5-55$ ) & $12.56(3.81)$ & $12.35(3.53)$ & $12.72(4.00)$ \\
\hline Dead & 36.7 & 44.9 & 30.1 \\
\hline Attrition & 21.0 & 15.6 & 25.4 \\
\hline Body mass index & $22.26(3.24)$ & $22.06(2.95)$ & $22.42(3.44)$ \\
\hline Underweight (less than 18.5) & 10.7 & 10.3 & 11.1 \\
\hline Normal (18.5-25.0) & 71.7 & 75.5 & 68.7 \\
\hline Overweight (more than 25.0) & 17.6 & 14.3 & 20.2 \\
\hline Smoking & 24.5 & 45.1 & 7.9 \\
\hline Alcohol use & 40.3 & 63.8 & 21.3 \\
\hline \multicolumn{4}{|l|}{ Physical activity } \\
\hline Act & 38.6 & 41.6 & 36.1 \\
\hline Moderate & 33.6 & 31.7 & 35.2 \\
\hline Non-active & 27.8 & 26.7 & 28.8 \\
\hline
\end{tabular}

Values represent $\%$ or mean (standard deviation). 
Table 2. Variation in body mass index and health behaviors by wave (1987-2006)

\begin{tabular}{|c|c|c|c|c|c|c|c|c|}
\hline$=$ & $\begin{array}{l}\text { Wave } 1 \\
(1987)\end{array}$ & $\begin{array}{l}\text { Wave } 2 \\
(1990)\end{array}$ & $\begin{array}{l}\text { Wave } 3 \\
(1993)\end{array}$ & $\begin{array}{l}\text { Wave } 4 \\
(1996)\end{array}$ & $\begin{array}{l}\text { Wave } 5 \\
\text { (1999) }\end{array}$ & $\begin{array}{l}\text { Wave } 6 \\
(2002)\end{array}$ & $\begin{array}{l}\text { Wave } 7 \\
(2006)\end{array}$ & Total \\
\hline Total participants at each & 2,200 & 2,037 & 1,841 & 2,429 & 3,424 & 2,718 & 2,020 & 16,669 \\
\hline Body mass index & $21.94(3.25)$ & $21.98(3.14)$ & $22.01(3.24)$ & $22.29(3.21)$ & $22.30(3.31)$ & $22.23(3.31)$ & $22.22(3.37)$ & $22.16(3.27)$ \\
\hline Smoking & $0.28(0.45)$ & $0.27(0.44)$ & $0.24(0.43)$ & $0.22(0.42)$ & $0.18(0.39)$ & $0.15(0.35)$ & $0.10(0.30)$ & $0.20(0.40)$ \\
\hline $1=$ yes & 28.2 & 27.0 & 24.0 & 22.4 & 18.2 & 14.6 & 10.2 & 20.3 \\
\hline $0=$ no & 71.8 & 73.0 & 76.0 & 77.6 & 81.8 & 85.4 & 89.8 & 79.7 \\
\hline Change in smoking (delta) & & $-0.01(0.26)$ & $-0.02(0.25)$ & $-0.02(0.22)$ & $-0.03(0.22)$ & $-0.03(0.22)$ & $-0.05(0.26)$ & $-0.03(0.24)$ \\
\hline $1=$ initiation & & 2.7 & 1.9 & 1.4 & 0.9 & 0.9 & 1.0 & 1.4 \\
\hline $0=$ no change & & 93.4 & 93.8 & 95.1 & 94.9 & 94.8 & 92.9 & 94.2 \\
\hline$-1=$ cessation & & 3.9 & 4.3 & 3.6 & 4.2 & 4.2 & 6.1 & 4.4 \\
\hline Alcohol use & $0.38(0.48)$ & $0.39(0.49)$ & $0.37(0.48)$ & $0.40(0.49)$ & $0.36(0.48)$ & $0.35(0.48)$ & $0.32(0.47)$ & $0.37(0.48)$ \\
\hline $1=$ yes & 37.8 & 38.7 & 37.4 & 40.5 & 36.2 & 34.7 & 32.3 & 36.8 \\
\hline $0=$ no & 62.2 & 61.3 & 62.6 & 59.5 & 63.8 & 65.3 & 67.7 & 63.2 \\
\hline $\begin{array}{l}\text { Change in alcohol use } \\
\text { (delta) }\end{array}$ & & $-0.02(0.40)$ & $-0.01(0.38)$ & $-0.04(0.37)$ & $-0.04(0.37)$ & $-0.04(0.37)$ & $-0.05(0.38)$ & $-0.03(0.38)$ \\
\hline $1=$ initiation & & 7.0 & 6.9 & 4.7 & 4.9 & 5.1 & 4.7 & 5.5 \\
\hline $0=$ no change & & 84.3 & 85.3 & 86.2 & 85.9 & 86.3 & 85.6 & 85.6 \\
\hline$-1=$ cessation & & 8.7 & 7.8 & 9.1 & 9.3 & 8.6 & 9.7 & 8.9 \\
\hline Physical activity & $1.09(0.81)$ & $1.11(0.79)$ & $1.22(0.79)$ & $1.14(0.79)$ & $1.12(0.82)$ & $1.13(0.82)$ & $0.11(0.82)$ & $1.13(0.81)$ \\
\hline $2=$ active & 37.5 & 37.2 & 44.2 & 39.3 & 40.6 & 40.6 & 39.3 & 39.8 \\
\hline
\end{tabular}

This article is protected by copyright. All rights reserved. 


\begin{tabular}{|c|c|c|c|c|c|c|c|c|}
\hline $1=$ moderate & 34.2 & 36.6 & 33.5 & 35.4 & 30.6 & 31.6 & 32.1 & 33.2 \\
\hline $0=$ non-active & 28.3 & 26.2 & 22.3 & 25.3 & 28.7 & 27.8 & 28.6 & 27.0 \\
\hline ge in physical a & & $-0.02(0.73)$ & $0.07(0.70)$ & $-0.11(0.71)$ & $-0.03(0.72)$ & $-0.04(0.69)$ & $-0.04(0.70)$ & $-0.03(0.71)$ \\
\hline 1=initiation & & 25.5 & 28.0 & 20.2 & 24.1 & 22.3 & 22.4 & 23.7 \\
\hline $0=$ no change & & 47.3 & 51.0 & 48.7 & 48.6 & 51.7 & 51.1 & 49.9 \\
\hline$-1=$ cessation & & 27.2 & 21.1 & 31.1 & 27.3 & 25.9 & 26.5 & 26.4 \\
\hline
\end{tabular}

Values represent number, mean (standard deviation) or $\%$.

This article is protected by copyright. All rights reserved. 
Table 3. The effect of health behaviors on the trajectory of body mass index

\begin{tabular}{|c|c|c|c|c|c|c|c|c|c|c|}
\hline Fixed effect & \multicolumn{2}{|c|}{ Model 1} & \multicolumn{2}{|c|}{ Model 2} & \multicolumn{2}{|c|}{ Model 3} & \multicolumn{2}{|c|}{ Model 4} & \multicolumn{2}{|c|}{ Model 5} \\
\hline \multicolumn{11}{|l|}{ Time-varying } \\
\hline Lagged & -0.722 & $* * *$ & -0.730 & $* * *$ & -0.358 & $* * *$ & -0.266 & $* *$ & -0.359 & $* *$ \\
\hline Delta & -0.431 & *** & -0.441 & $* * *$ & -0.281 & ** & -0.231 & * & -0.298 & * \\
\hline Lagged $\times$ Baseline age & & & & & & & 0.040 & ** & & \\
\hline Delta $\times$ Baseline age & & & & & & & 0.020 & & & \\
\hline Lagged $\times$ Female & & & & & & & & & -0.006 & \\
\hline Delta $\times$ Female & & & & & & & & & -0.063 & \\
\hline \multicolumn{11}{|l|}{ Drinking } \\
\hline Lagged & 0.137 & & 0.066 & & 0.038 & & 0.004 & & 0.040 & \\
\hline Delta & 0.154 & $*$ & 0.108 & & 0.096 & & 0.103 & & 0.097 & \\
\hline Lagged $\times$ Baseline age & & & & & & & -0.016 & & & \\
\hline Delta $\times$ Baseline age & & & & & & & 0.001 & & & \\
\hline Lagged $\times$ Female & & & & & & & & & 0.035 & \\
\hline Delta $\times$ Female & & & & & & & & & 0.032 & \\
\hline \multicolumn{11}{|l|}{ Physical activity } \\
\hline Lagged & 0.075 & & 0.020 & & 0.022 & & 0.020 & & 0.025 & \\
\hline Delta & 0.067 & $*$ & 0.022 & & 0.019 & & 0.030 & & 0.023 & \\
\hline Lagged $\times$ Baseline age & & & & & & & -0.002 & & & \\
\hline Delta $\times$ Baseline age & & & & & & & 0.003 & & & \\
\hline
\end{tabular}

This article is protected by copyright. All rights reserved. 
Lagged $\times$ Female

Delta $\times$ Female

Other covariates

Married (lagged)

Married (delta)

Current working (lagged)

Current working (delta)

Household income (lagged)

Household income (delta)

Total condition (lagged)

Total condition (delta)

Self-rated health (lagged)

Self-rated health (delta)

Functional capacity (lagged)

Functional capacity (delta)

Time-constant

For intercept

Intercept
Female
Age
Education
Dead
Attrition
Baseline body mass index

Baseline body mass index
0.123

0.179

0.023

$-0.018$

0.053

0.047

0.074

0.044 *

$-0.067^{* * *}$

$-0.055^{* * *}$

$-0.028$

$-0.012$

$$
\begin{array}{r}
21.9988^{* * *} \\
0.022 \\
-0.079 \\
-0.041^{* * *} \\
-0.957 \\
-0.028
\end{array}
$$

$22.010{ }^{* * *}$
0.081
-0.073
$-0.047 \quad * * *$
-0.891
-0.004

$-0.1299^{*}$
$-0.144^{*}$
-0.081
0.094
0.061
0.015
0.051
0.047
0.023
0.022
-0.052
-0.052
-0.020
-0.012

$\begin{array}{rrrrr}22.016^{* * *} & 22.019^{* * *} & 22.016^{* * *} \\ -0.083 & & -0.089 & -0.082 & \\ -0.031^{* * *} & -0.031^{* * *} & -0.032^{* * *} \\ -0.041^{* *} & -0.042^{* *} & -0.042^{* *} \\ -0.416^{* * *} & -0.421^{* * *} & -0.416^{* * *} \\ 0.093^{* * *} & 0.093^{* * *} & 0.094^{*} & \\ 0.755^{* * *} & 0.754^{* * *} & 0.755^{* * *}\end{array}$

This article is protected by copyright. All rights reserved. 
For linear slope

Intercept
Female
Age
Education
Dead
Attrition

\begin{tabular}{|c|c|c|}
\hline-0.054 & **** & -0.054 \\
\hline 0.012 & & 0.012 \\
\hline-0.003 & * & -0.003 \\
\hline-0.002 & & -0.002 \\
\hline-0.039 & $*$ & -0.039 \\
\hline 0.005 & & 0.005 \\
\hline
\end{tabular}

Baseline body mass index

For quadratic slope

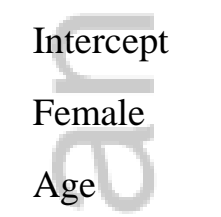

Education

Attrition

Baseline body mass index

Random effect (variance component)

\begin{tabular}{lrrrrrr} 
Intercept & $7.937^{* * *}$ & $7.892^{* * *}$ & $2.493^{* * *}$ & $2.482^{* * *}$ & $2.491^{* * *}$ \\
Linear slope & $0.038^{* * *}$ & $0.038^{* * *}$ & $0.036^{* * *}$ & $0.035^{* * *}$ & $0.036^{* * *}$ \\
\hline Quadratic slope & $0.0002^{*}$ & $0.0002^{* *}$ & $0.0002^{* *}$ & $0.0002^{*}$ & $0.0002^{*}$ \\
Level 1 & 2.162 & 2.153 & 2.179 & 2.180 & 2.177 \\
\hline
\end{tabular}

${ }^{* * *}: \mathrm{p}<0.001 .^{* *}: \mathrm{p}<0.01 .{ }^{*}: \mathrm{p}<0.05$.

This article is protected by copyright. All rights reserved. 